\title{
Neurite Outgrowth in Individual Neurons of a Neuronal Population Is Differentially Regulated by Calcium and Cyclic AMP
}

\author{
Mark P. Mattson, Anne Taylor-Hunter, and Stanley B. Kater \\ Program in Neuronal Growth and Development, Department of Anatomy and Neurobiology, Colorado State University, \\ Fort Collins, Colorado 80523
}

In identified Helisoma neurons, intracellular calcium can regulate neurite elongation and growth cone motility. Neurotransmitters such as 5-HT suppress both neurite elongation and the filopodial and lamellipodial movements of growth cones by causing increases in intracellular calcium (Haydon et al., 1984; Cohan et al., 1987; Mattson and Kater, 1987). Since an additional second messenger, cyclic AMP (CAMP), is known to mediate many physiological effects of neurotransmitters, we tested (1) the possible involvement of CAMP in the regulation of neurite outgrowth from Helisoma buccal neurons and (2) calcium-cAMP interrelationships in the regulation of outgrowth. The cAMP-elevating agents forskolin $\left(5 \times 10^{-6}-10^{-4} \mathrm{M}\right)$ and dibutyryl CAMP (dbcAMP; $5 \times 10^{-3}-$ $10^{-2} \mathrm{M}$ ) suppressed neurite elongation and growth cone movements in identified neurons B19 (5-HT sensitive) and B5 (5-HT insensitive); the suppression was reversible. Exposure of these particular identified neurons to the calcium channel blocker $\mathrm{La}^{3+}\left(10^{-5} \mathrm{M}\right)$ or a culture medium with reduced calcium prevented and reversed the suppressive effects of forskolin and dbcAMP. In order to determine if the results on neurons $B 5$ and $B 19$ were representative of all neurons or only a subset, we examined a larger population of neurons. Calcium ionophore A23187 suppressed outgrowth from all neurons in mass dissociate cultures of buccal neurons, while forskolin or dbcAMP plus IBMX suppressed outgrowth from only one-half of buccal neurons. Finally, we found that 2 subpopulations exist among the neurons whose outgrowth is suppressed by CAMP: One subpopulation requires calcium influx for CAMP to act, while the other does not. Thus, even within the relatively small population of neuronal types comprising the buccal ganglion of Helisoma, second messengers within different neurons can act and interact in different ways to regulate outgrowth.

Various signals-including growth factors, neurotransmitters, and electrical activity - are known to affect neuronal outgrowth and are therefore likely to be important regulators of the formation and modulation of neuronal architecture. We are currently investigating the intracellular mechanisms by which these signals act to alter growth cone motility and neurite elongation by studying the behavior of identified neurons from the snail

\footnotetext{
Rccived July 9, 1987; revised Sept. 18, 1987 ; accepted Oct. 5, 1987.

We acknowledge the technical assistance of Michelle Mischke, Denise Dehnbostel, and Lisa Spirio. We thank Drs. L. Mills and M. Schmidt for their critical comments on the manuscript. This work was supported by NIH Grants NS 08054 (M.P.M.), NS 24683, NS 24561, and NS 15350 (S.B.K.), and a gift from the Monsanto Corporation.

Correspondence should be addressed to Dr. Mattson at the above address. Copyright (C) 1988 Society for Neuroscience $0270-6474 / 88 / 051704-08 \$ 02.00 / 0$
}

Helisoma in culture. Second messengers-including cyclic nucleotides, calcium, and inositol phospholipids-are prime candidates as they are known to regulate an array of biochemical processes in a variety of cells (Sutherland, 1972; Greengard, 1978; Berridge, 1984; Nishizuka, 1984). Calcium and cAMP systems often interact to regulate ccll functions, but the nature of the interactions varies depending upon the cell type (Rasmussen and Barrett, 1984). For example, cAMP increases calcium influx in several neuronal types (Schubert et al., 1978; Connor and Hockberger, 1985), while calcium can reduce intracellular cAMP in some endocrine tissues (Rasmussen and Barrett, 1984; Mattson and Spaziani, 1986). Pertinent to the regulation of neuronal outgrowth, it has been shown that manipulations of cAMP (Shapiro, 1973; Schubert et al., 1978; Nirenberg et al., 1984), calcium (Letourneau and Wessels, 1974; Anglister et al., 1982; Kostenko et al., 1983; Bixby and Spitzer, 1984; Suarez-Isla et al., 1984; Cohan et al., 1987; Mattson and Kater, 1987), and/or protein kinase C (Ishii, 1978; Reboulleau, 1986) can stimulate or suppress neurite outgrowth. However, these effects have not been examined in detail at the levels of the single identified neuron or growth cone, nor has the generality of second messenger involvement in neuronal outgrowth among the diverse neuronal types comprising a nervous system been tested.

Considerable information on the regulation of neurite outgrowth has been obtained from studies of isolated identified neurons from the mollusc Helisoma (see Kater, 1985; Kater and Mattson, 1987, for reviews). In addition to spontaneous conditions of growth or stabilization, outgrowth of these neurons can be affected by physiologically relevant stimuli, including neurotransmitters and electrical activity. Neurotransmitters suppress outgrowth in a neuron-specific manner (Haydon et al., 1984, 1985; McCobb et al., 1985; Goldberg et al., 1986) while electrical activity suppresses outgrowth in all neurons tested (Cohan et al., 1985; Cohan and Kater, 1986). Regulation of both growth cone motility and neurite elongation by neurotransmitters requires influx of extracellular calcium, and we have recently provided evidence that there are different optimal levels of calcium influx that promote growth cone movements and neurite elongation (Cohan et al., 1987; Mattson and Kater, 1987). Furthermore, our results showed that a single intracellular molecule, calcium, can regulate the expression of a variety of neuronal morphologies in different identified neurons. Thus, a neurite may elongate, elongate and branch, stop, or stop and go depending upon changes in calcium influx (Mattson and Kater, 1987).

The collective data on calcium involvement in the regulation of neurite outgrowth in Helisoma and the facts that cAMP me- 
diates many of the cellular actions of neurotransmitters (Greengard, 1978) and can affect cellular calcium-regulating systems (Kaczmarek et al., 1980; Doroshenko et al., 1984) prompted us to design experiments to determine (1) if $\mathrm{c} \Lambda \mathrm{MP}$ alters neurite outgrowth in identified Helisoma neurons, (2) if and how calcium and cAMP systems interact in regulating outgrowth, and (3) if calcium and/or cAMP are general regulators of neuronal outgrowth affecting all neurons in a population or if they are active in only a particular subset of the population. The data reported here suggest that calcium is a general regulator of neuronal outgrowth in Helisoma buccal neurons, while cAMP acts only upon a subset of neurons. Furthermore, there is an additional subpopulation of neurons among those whose outgrowth is sensitive to cAMP that require influx of calcium for suppression of outgrowth.

\section{Materials and Methods}

Animal dissection and neuronal culture. Helisoma trivolvis (red) snails with shell diameters of $10-18 \mathrm{~mm}$ were maintained as previously described (Haydon et al., 1985). Removal of the buccal ganglia was done under sterile conditions (Wong et al., 1981). Ganglia were incubated for 45-60 min in culture medium (HL-15; 50\% Leibowitz L-15 modified for Helisoma; see Wong et al., 1981) containing 0.1\% trypsin (Type III$\mathrm{S}$, Sigma) followed by a 10 min incubation in HL-15 containing $0.1 \%$ trypsin inhibitor (Sigma). Ganglia were pinned dorsal side up to Sylgard pads. Identified neurons (B19 and B5), visualized under a dissecting microscope, were removed by first making an incision in the ganglionic sheath dorsolateral to the neuronal somata and then gently sucking the neuron into a micropipette containing HL-15. Isolated neurons were transferred to culture dishes with polylysine-coated glass or plastic substratum and containing $2 \mathrm{ml} \mathrm{HL}-15$ conditioned with Helisoma brains (conditioned medium contains a factor that associates with the substratum and promotes extensive neurite outgrowth; Wong et al., 1981, 1984). For mass dissociation of buccal neurons, ganglia were prepared for cell removal as described above; a large slit was then made on the dorsal aspect of the ganglionic sheath, and the entire ganglionic contents were removed to culture dishes (prepared and conditioned as described above) with the aid of a micropipette. Cells were dissociated by repeated trituration. Typically, these cultures contained from 30-60 neurons. Neurons were cultured in a humidified room air atmosphere at 22$25^{\circ} \mathrm{C}$.

Treatment of cultured neurons. The adenylate cyclase activator forskolin (Daly et al., 1982) and the calcium ionophore A23187 (Reed and Lardy, 1972) were dissolved in dimethylsulfoxide (DMSO) and were added to cultures in 5-10 $\mu 1$ volumes (DMSO alone at these levels was added to control plates and did not affect neuronal outgrowth). $\mathrm{LaCl}_{3}$, $\mathrm{N}^{6}, \mathrm{O}^{2}$-dibutyryladenosine- $3^{\prime}: 5^{\prime}$-cyclic monophosphate (dbcAMP), and 3-isobutyl-1-methylxanthine (IBMX) were dissolved in HL-15 and were added to cultures in 22-220 $\mu \mathrm{l}$ volumes. Concentrations reported are the final concentration in culture medium. All agents were from Sigma Chemical Co. (St. Louis). Reduced calcium medium consisted of HL15 in which $\mathrm{MgCl}_{2}$ was substituted for $\mathrm{CaCl}_{2}$. Growth cones were isolated by severing the neurite just proximal to the growth cone using an electrolytically sharpened tungsten microknife.

Assessments of neurite elongation rate and growth cone motility. For assessments of neurite elongation, cells and isolated growth cones were examined and photographed with the aid of a Nikon Diaphot inverted microscope with phase-contrast optics. Neurite elongation rates were quantified using tracings made from projected negatives of photographs taken at $30 \mathrm{~min}$ intervals. Linear-regression analysis of pre- and posttreatment elongation rates was used to obtain the post/pretreatment ratio, which is expressed as percentage of the initial elongation rate. Growth cone motility was examined either from projected negatives or from video images (e.g., Fig. 3). The video system consisted of a Zeiss ICM-35 microscope (phase-contrast, $100 \mathrm{X}$ oil-immesion objective), an RCA SIT (silicon intensifier target) camera, and a Quantex image analysis system. Photographs were taken directly from the video screen. The number of filopodia/growth cone was used as a measure of growth cone motility since these structures are known to reflect the underlying activity of the growth cone cytoskeleton (Bray and Gilbert, 1981; cf. Mattson and Kater, 1987). Analysis of the effects of experimental treat-

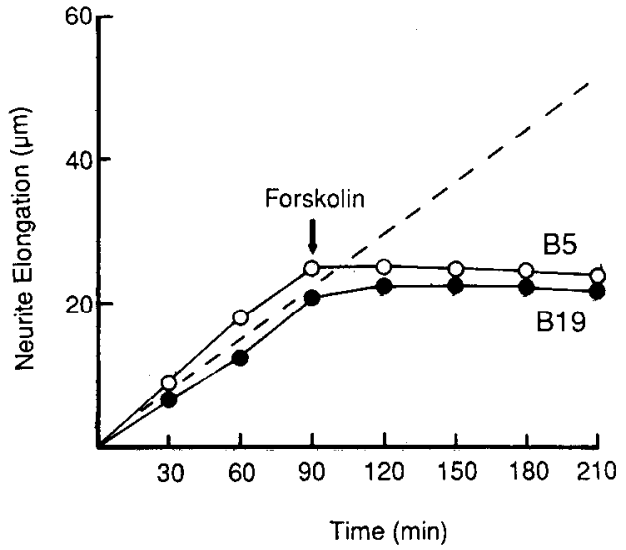

Figure 1. Time course of the suppressive effect of forskolin on neurite elongation. Neurons B19 (solid circles) and B5 (open circles) were preincubated $90 \mathrm{~min}$ in the absence of forskolin after which forskolin $\left(10^{-5}\right.$ $M$ final concentration) was added and incubations were continued an additional $2 \mathrm{hr}$; neurite elongation was determined from photographs taken at $30 \mathrm{~min}$ time interevals. The values presented for forskolintreated neurons are from individual neurites and are representative of the effects seen on all of 12 B19 and 17 B5 neurites (3-5 neurons). The dashed line represents the elongation rate of neurites from control neurons (average from 27 neurites)

ments on outgrowth from generic buccal neurons in mass dissociates employed video images of neurons taken $6 \mathrm{hr}$ postplating and immediately prior to treatment, and $4 \mathrm{hr}$ posttreatment. Both growth cone motility and neurite elongation were examined in the generic neurons. Outgrowth was scored as stabilized if all neurites possessed phase-bright distal tips and if neurite elongation had ceased. In all cascs cxamincd (over 500 neurites), the appearance of a phase-bright neurite tip (i.e., retraction of growth cone filopodia and lamellipodia) coincided with cessation of neurite elongation (see Haydon et al., 1984). The number of stabilized neurons at $4 \mathrm{hr}$ posttreatment was expressed as a percentage of the total neurons examined/dish. Student's $t$ test was used for all statistical comparisons, and values are expressed as means and SEM.

\section{Results}

CAMP and calcium regulation of neurite outgrowth from identified neurons

cAMP-related suppression of neurite outgrowth

Our initial experiments tested the possible involvement of cAMP in the regulation of neurite outgrowth. We measured the effects on neurite elongation and growth cone motility of several agents known to increase intracellular cAMP. The adenylate cyclase activator forskolin $\left(10^{-5} \mathrm{M}\right)$ rapidly (within $30 \mathrm{~min}$ of exposure) inhibited neurite elongation in identified neurons B5 and B19 (Fig. 1). The suppressive effect of forskolin was dose dependent within the concentration range of $10^{-6}-10^{-4} \mathrm{M}$; the $\mathrm{ED}_{50}$ for suppression was approximately $5 \times 10^{-6} \mathrm{M}$ (Fig. 2). Suppression of neurite elongation by forskolin was reversible. Neurites (15 neuron B5 neurites and 12 B19 neurites) elongating at an average rate of $18.5 \pm 4.7 \mu \mathrm{m} / \mathrm{hr}$ prior to exposure to forskolin were suppressed during a $60 \mathrm{~min}$ exposure to $10^{-5} \mathrm{M}$ forskolin (rate was reduced to $1.1 \pm 3.6 \mu \mathrm{m} / \mathrm{hr}$ ). Culture dishes were then washed with fresh medium, and elongation resumed at a rate similar to the pretreatment rate $(16.6 \pm 4.1 \mu \mathrm{m} / \mathrm{hr}$; see Fig. 3$)$. An additional test for cAMP involvement was to employ the membrane-permeant cAMP analog dbcAMP and the phosphodiesterase inhibitor IBMX. Again, neurite elongation was suppressed in a dose-dependent manner by dbcAMP $\left(10^{-3}-10^{-2}\right.$ M; Fig. 2), but complete suppression with this agent was not seen until 60 min posttreatment, possibly due to a lag in pen- 


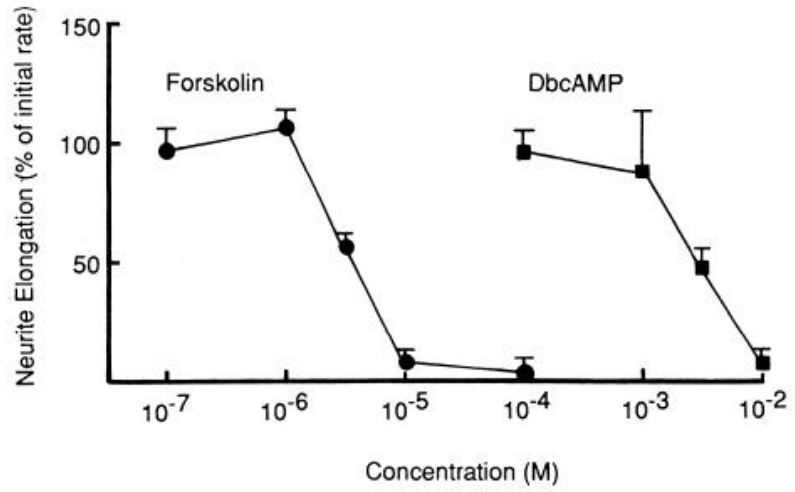

Figure 2. Concentration dependence of the suppressive effects of forskolin and dbcAMP on neurite elongation. Elongation rates of neurites from neurons B19 and B5 (values represent combined data) were determined during a $90 \mathrm{~min}$ preincubation to establish the initial (pretreatment) rate of neurite elongation; neurons were then exposed to either forskolin or dbcAMP at the indicated concentrations, and elongation rates were determined for a $2 \mathrm{hr}$ posttreatment period. Values represent the mean and SEM of determinations on 7-19 neurites from 3-7 neurons. Suppression was significant compared to controls for forskolin concentrations of $5 \times 10^{-6}$ or greater and for dbcAMP concentrations of $5 \times 10^{-3}$ or greater $(p<0.01-0.001)$. Outgrowth of control neurites averaged $106.4 \pm 7.1 \%$ of the initial rate.

etration of this compound into the cytoplasm. In combination with $10^{-4} \mathrm{M}$ IBMX, dbcAMP was effective at $10^{-4} \mathrm{M}$ (Fig. 5).

Growth cone motility as evidenced by filopodial structures is reduced in neurons exposed to inhibitory signals, including neurotransmitters, electrical activity, and ionophore A23187 (Haydon et al., 1985; Cohan et al., 1987; Mattson and Kater, 1987). This same pattern of effects was seen in neurons B19 and B5 exposed to forskolin or dbcAMP (Fig. 3, Table 1). Filopodia were greatly reduced in number in response to the cAMP-elevating agents and neurite elongation was suppressed (Fig. 3). This suppression was reversible as filopodia and lamellipodia reformed, and elongation continued following washout of the forskolin (Fig. 3).

Previous studies demonstrated that suppression of outgrowth can be mediated directly by the growth cone (Haydon et al., 1985; Mattson and Kater, 1987). cAMP also appears to act within the growth cone proper since exposure of isolated growth cones to forskolin or dbcAMP resulted in a significant reduction

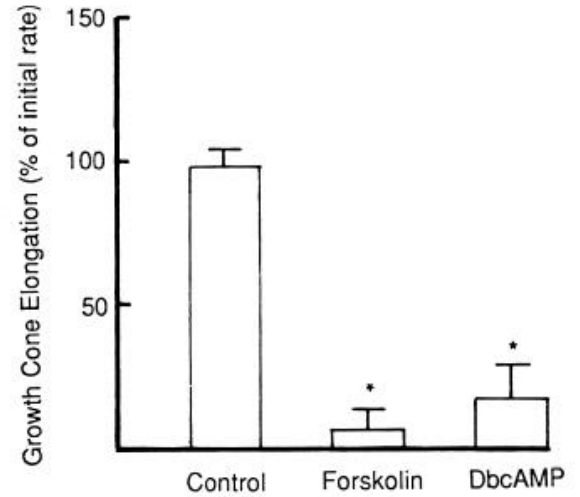

Figure 4. Effects of forskolin and dbcAMP on the elongation of isolated growth cones. Isolated growth cones from neurons B5 and B19 (data combined) were exposed to forskolin $\left(10^{-5} \mathrm{M}\right)$ or dbcAMP $\left(10^{-2}\right.$ M) following an initial 90 min postisolation incubation period, which was used to determine the initial rate of elongation; elongation was determined for a subsequent $2 \mathrm{hr}$ period. Values represent the mean and SEM of determinations on 3-7 isolated growth cones. ${ }^{*} p<0.001$ compared to control.

in elongation rate (Fig. 4). Elongation was significantly reduced to 7 and $18 \%$ of control rates by forskolin $\left(10^{-5} \mathrm{M}\right)$ and by dbcAMP $\left(10^{-2} \mathrm{M}\right)$, respectively (Fig. 4). The inhibition of elongation was accompanied by a reduction in growth cone filopodia and lamellipodia, as was seen for intact neurites (cf. Fig. 3). These results demonstrate that, at least in cultured neurons, growth cone motility and elongation can be regulated autonomously by increases in cAMP.

\section{CAMP-calcium interactions in the regulation of neurite outgrowth}

In many cell types calcium influx across the plasma membrane can be regulated by cAMP (Connor and Hockberger, 1985; Levitan, 1985). Furthermore, it is clear that in different cell types calcium and cAMP systems interact in different ways to bring about a cell response (Rasmussen and Barrett, 1984). Since changes in calcium influx can regulate neurite outgrowth in Helisoma neurons, we reasoned that the cAMP and calcium systems might interact to regulate neurite outgrowth. We therefore tested the calcium dependency of cAMP's effects on outgrowth from neurons B5 and B19 by exposing these neurons to combined
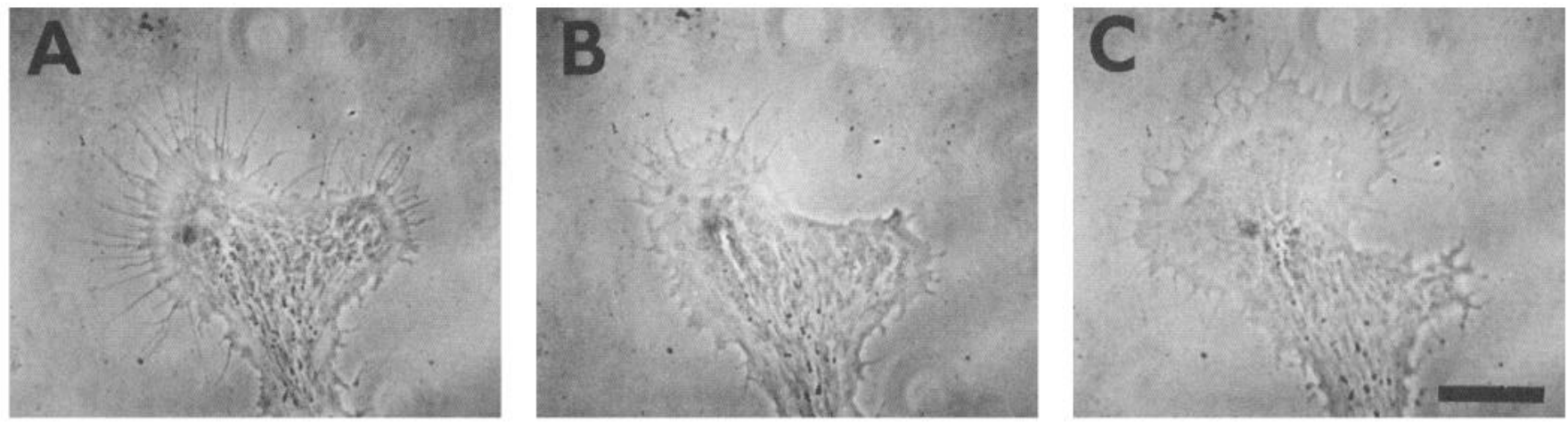

Figure 3. Reversibility of the suppressive effect of forskolin on growth cone motility. Computer-digitized video images of a growth cone of a neuron B5 immediately prior to treatment with $10^{-5} \mathrm{M}$ forskolin $(A), 20 \mathrm{~min}$ posttreatment $(B)$, and 50 min after washout of the forskolin (four 5 min washes in fresh HL-15 medium $(C)$. Note that forskolin caused a reduction in the number of filopodia and that filopodial activity increased following washout of the forskolin. These morphological effects were seen in all 43 growth cones examined. Calibration, $10 \mu \mathrm{m}$. 


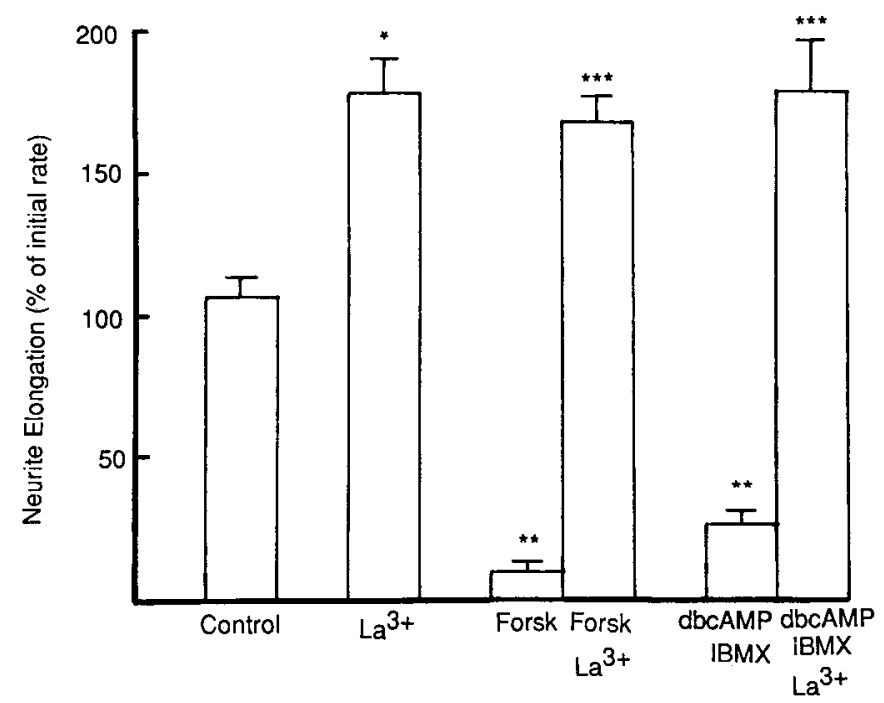

Figure 5. Effect of combined treatments with $\mathrm{I}^{3+}$ and either forskolin or dbcAMP-IBMX on neurite elongation. Abbreviations and concentrations were: $\mathrm{La}^{3+}, \mathrm{LaCl}_{2}\left(10^{-5} \mathrm{M}\right)$; Forsk, forskolin $\left(10^{-5} \mathrm{M}\right)$; dbcAMP $\left(10^{-3} \mathrm{M}\right)$; IBMX $\left(10^{-4} \mathrm{M}\right)$. Bars represent the mean and SEM of determinations on 9-12 neurites from 3-5 neurons (data from neurons B19 and $\mathrm{B} 5$ are combined). ${ }^{*} p<0.001$ compared to control; ${ }^{* *} p<0.01-$ 0.001 compared to control; *** $p<0.05-0.01$ compared to control, $p<$ 0.001 compared to forskolin or dbcAMP-IBMX.

treatments with forskolin and either the calcium channel blocker $\mathrm{La}^{3+}$ or culture medium with a reduced calcium concentration (Figs. 5-7).

The calcium channel blocker $\mathrm{La}^{3+}\left(10^{-5} \mathrm{M}\right)$, as reported previously (Mattson and Kater, 1987), both accelerated neurite elongation (to $178 \%$ of control levels; Fig. 5) and suppressed growth cone motility (see Fig. 7). Forskolin $\left(10^{5} \mathbf{M}\right)$ or the combination of dbcAMP $\left(10^{-3} \mathrm{M}\right)$ and IBMX $\left(10^{-4} \mathrm{M}\right)$ suppressed elongation (to $15-25 \%$ of control levels; Fig. 5) and growth cone motility (Fig. 3, Table 1). When neurons were exposed to combined treatments with forskolin and $\mathrm{La}^{3+}$, or dbcAMP-IBMX and $\mathrm{La}^{3+}$, the rates of neurite elongation were similar to those seen with $\mathrm{La}^{3+}$ treatment alone $(168 \%$ of controls; Fig. 5). The prevention of the suppressive effect of forskolin by $\mathrm{La}^{3+}$ was also seen when neurons were exposed to $\mathrm{La}^{3+} 30 \mathrm{~min}$ prior to treatment with forskolin (Fig. 6). Moreover, $\mathrm{La}^{3+}$ was able to reverse the suppression of both neurite elongation (Fig. 6) and growth cone motility (Fig. 7) caused by exposure to forskolin; this rescue effect was evident by $60 \mathrm{~min}$ following exposure to $\mathrm{La}^{3+}$ (Fig. 6). Following addition of $\mathrm{La}^{3+}$ to the inhibited neurites, elongation resumed, but growth cone filopodial and lamellipodial activity did not resume (Fig. 7). These results suggested that in neurons B5 and B19, influx of calcium across the plasma membrane is required for cAMP to exert its suppressive effect on outgrowth. As a further test of the calcium dependence of cAMP's action, we examined the effects of combined manipulations of extracellular calcium levels and cAMP.

In order to reduce the levels of extracellular calcium, the normal culture medium containing $4.1 \mathrm{~mm}$ calcium was replaced with medium containing reduced calcium (nominally zero). Controls consisted of cultures subjected to a medium change to normal defined HL-15 medium; as previously reported (Mattson and Kater, 1987), this control medium change alone enhanced neurite elongation rates (Fig. 8). As was seen

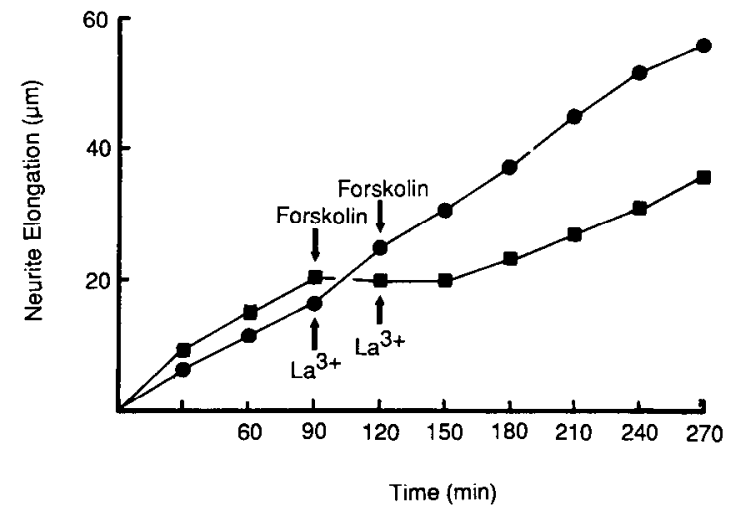

Figure 6. $\mathrm{La}^{3+}$ reverses suppression of neurite outgrowth induced by forskolin. The graph depicts the time course of neurite elongation of individual neurites from neurons B5 given staggered treatments with forskolin $\left(10^{-5} \mathrm{M}\right)$ or $\mathrm{La}^{3+}\left(10^{-5} \mathrm{M}\right)$ at the times indicated by the arrows. These effects were seen on 7 of 7 neurites from 3 neurons B5 and 10 of 10 neurites from 4 neurons B19.

above with the calcium channel blocker $\mathrm{La}^{3+}$, neurite elongation was significantly accelerated in the reduced calcium environment to $140 \%$ of control levels (Fig. 8). Forskolin $\left(10^{-5} \mathbf{~ M}\right)$ significantly reduced the elongation rates of neurons incubated in medium with physiological levels of calcium to $17 \%$ of the control level. Howcver, forskolin was incffectivc in suppressing outgrowth in the reduced extracellular calcium environment (Fig. 8). The effects of the reduced calcium environment on growth cone motility was qualitatively similar to that seen with $\mathrm{La}^{3+}$ (Fig. 7). Thus, as with 5-HT suppression of neurite outgrowth (Mattson and Kater, 1987), inhibition by cAMP requires influx of calcium from the cell exterior.

\section{cAMP and calcium regulation of neurite outgrowth from the total population of neurons in the buccal ganglia}

While neurotransmitters affect neurite outgrowth in a neuronspecific manner (Haydon et al., 1985), calcium, and cAMP might suppress outgrowth from all neurons nonselectively. Indeed, the fact that calcium- or cAMP-elevating agents suppressed neurite outgrowth from both the 5-HT-sensitive neuron B19 and the insensitive neuron B5 was consistent with this hypothesis. In order to test a more global role for cAMP and/or calcium in regulating neurite outgrowth, we examined a larger population, namely, the neurons of the buccal ganglia. We exposed cultured, buccal neurons plated as mass dissociates to cAMP-elevating

Table 1. Effects of cAMP-elevating agents on growth cone filopodia

\begin{tabular}{llll} 
& & \multicolumn{2}{l}{ Filopodia/growth cone } \\
\cline { 2 - 4 } Treatment & Neuron & $\begin{array}{l}\text { Before } \\
\text { treatment }\end{array}$ & $\begin{array}{l}\text { After } \\
\text { treatment }\end{array}$ \\
\hline Forskolin $\left(10^{-4} \mathrm{M}\right)$ & $\mathrm{B} 5$ & $20.0 \pm 2.3^{a}$ & $2.2 \pm 0.6^{b}$ \\
& $\mathrm{~B} 19$ & $8.0 \pm 0.7$ & $0.8 \pm 0.4^{b}$ \\
DbcAMP $\left(10^{-3} \mathrm{M}\right)$ & $\mathrm{B} 5$ & $17.4 \pm 1.8$ & $2.2 \pm 0.5^{b}$ \\
+ IBMX $\left(10^{-4} \mathrm{M}\right)$ & $\mathrm{B} 19$ & $7.0 \pm 0.7$ & $1.2 \pm 0.4^{b}$
\end{tabular}

Values represent the mean \pm SEM of determinations on 5 growth cones. Filopodial counts were made immediately prior to treatment and 60 min after treatment. a Growth cones of neurons B5 possess significantly more filopodia than do B19 growth cones $(p<0.01$; cf. Haydon et al., 1985).

${ }^{b} p<0.01-0.001$ compared to "Before treatment" values. 

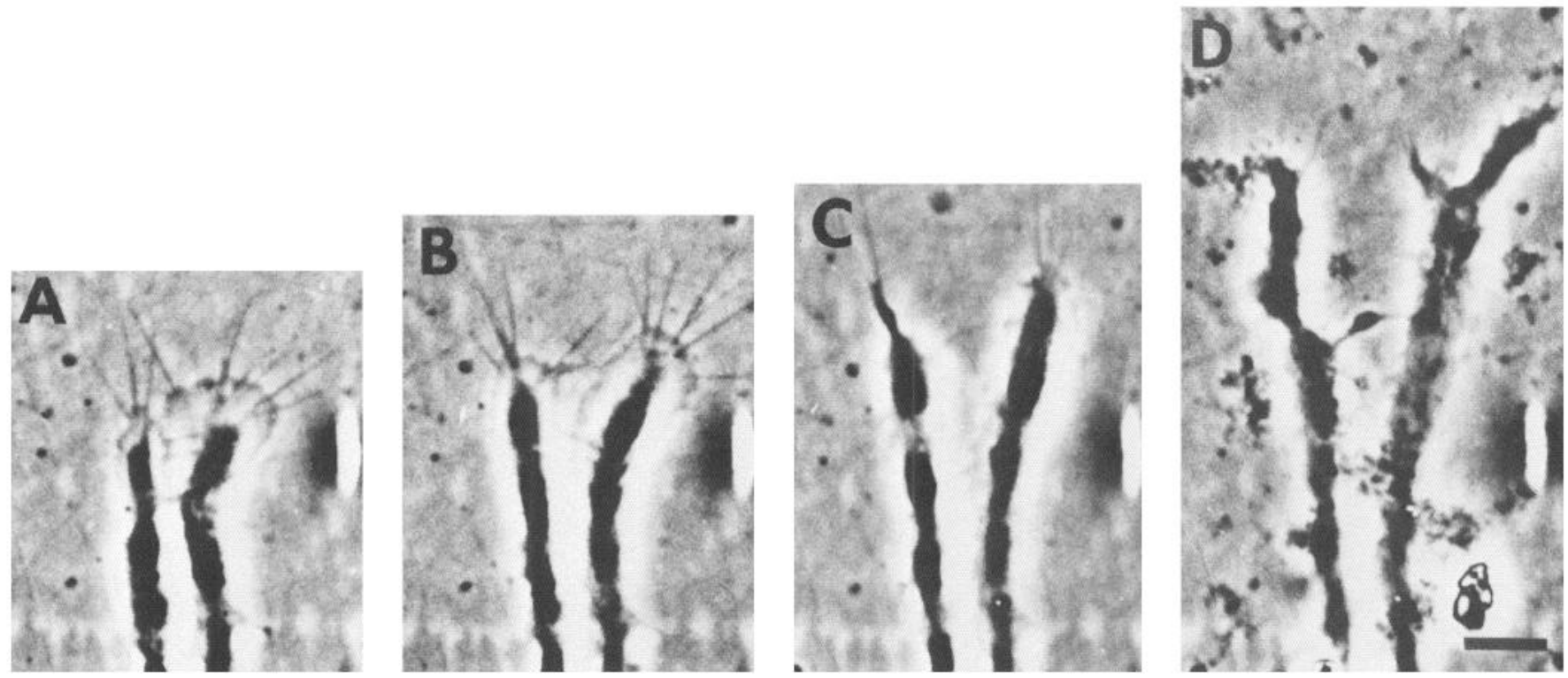

Figure 7. Effects of successive treatments with forskolin and $\mathrm{La}^{3+}$ on growth cone motility. $A$ and $B$, Photomicrographs of 2 neuron $\mathrm{B} 19$ growth cones with multiple, active filopodia and broad lamellipodia $30 \mathrm{~min}(A)$ and immediately $(B)$ prior to treatment with forskolin. $C$, The same growth cones $60 \mathrm{~min}$ following exposure to forskolin $\left(10^{-5} \mathrm{M}\right)$ and immediately prior to treatment with $\mathrm{La}^{3+}$; note that filopodia are greatly reduced in numbers and the lamellipodia have retracted leaving a neurite-width distal tip. $D$, Sixty minutes following treatment with $\mathrm{La}^{3+}\left(10^{-5} \mathrm{M}^{3}\right.$ in the continued presence of forskolin; the neurites have continued to elongate, but growth cone motility is still reduced. This series of morphological hanges was seen in all 27 growth cones examined. Calibration, $10 \mu \mathrm{m}$.

agents, as well as to treatments known to increase or reduce calcium influx (Table 2). The outgrowth of such neurons could then be assessed in terms of neurite elongation and growth cone motility (see Materials and Methods).

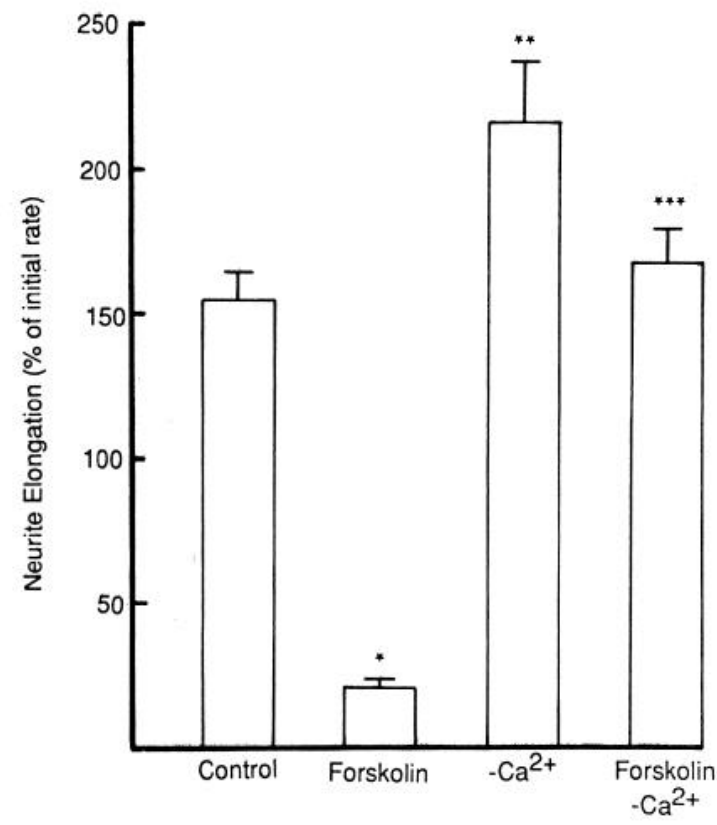

Figure 8. Effects of reduced extracellular calcium on neurite elongation as affected by forskolin. Following preincubations to determine initial neurite elongation rates, neurons $\mathrm{B} 19$ and $\mathrm{B} 5$ were exposed to fresh $\mathrm{HL}$ 15 medium (Control), $\mathrm{HL}-15$ with $\mathrm{MgCl}_{2}$ substituted for $\mathrm{CaCl}_{2}\left(-\mathrm{Ca}^{2+}\right)$; forskolin $\left(10^{-4} \mathrm{M}\right)$ in normal HL-15; or forskolin in HL-15 lacking $\mathrm{Ca}^{2+}$. Bars represent the mean and SEM of determinations from 8-11 neurites from 3-4 neurons (data from neurons B19 and B5 are combined). ${ }^{*} p<0.001$ compared to control; ${ }^{* *} p<0.01$ compared to control; *** $p<0.001$ compared to forskolin.
We previously reported that the calcium ionophore A23187 at $10^{-7} \mathrm{M}$ completely suppressed outgrowth from neurons B19 and B5 (Mattson and Kater, 1987). Similarly, we found here that neurite outgrowth from all buccal neurons was suppressed by $10^{-7} \mathrm{M} \mathrm{A} 23187$ (Table 2). This result suggested that calcium influx may be a general trigger for stabilization of growing neurons. Since cAMP-elevating agents also suppressed outgrowth from neurons B19 and B5 (Fig. 2), we next tested the generality of cAMP's action on the total population of buccal neurons (Table 2). Even when exposed to levels of forskolin $\left(10^{-4} \mathrm{M}\right)$ or the combination of dbcAMP $\left(10^{-3} \mathrm{M}\right)$ and IBMX $\left(10^{-4} \mathrm{M}\right)$ that completely stopped outgrowth in neurons B5 and B19, only

Table 2. Effects of cAMP- and calcium-altering agents upon neurite outgrowth from unidentified buccal neurons

\begin{tabular}{lc} 
Treatment & $\begin{array}{l}\text { Stable state neurons } \\
(\%)\end{array}$ \\
\hline Control & $7.0 \pm 1.8(210)$ \\
A23187 $\left(10^{-7} \mathrm{M}\right)$ & $98.1 \pm 2.3^{a}(50)$ \\
Forskolin $\left(10^{-4} \mathrm{M}\right)$ & $56.7 \pm 2.1^{b}(124)$ \\
dbcAMP $\left(10^{-3} \mathrm{M}\right)+\operatorname{IBMX}\left(10^{-4} \mathrm{M}\right)$ & $52.4 \pm 3.9^{b}(85)$ \\
Reduced $\left[\mathrm{Ca}^{2+}\right]_{\mathrm{e}}$ & $24.1 \pm 5.5^{c}(68)$ \\
Forskolin $\left(10^{-4} \mathrm{M}\right)+$ reduced $\left[\mathrm{Ca}^{2+}\right]_{\mathrm{e}}$ & $43.3 \pm 6.1^{d}(87)$
\end{tabular}

Neurons plated as mass dissociates were exposed to vehicle (Control) or the agents indicated six hours after plating. Outgrowth status was assessed four hours later and neurons with phase-bright, non-elongating neurites were scored as suppressed (see Materials and Methods). Values represent the mean \pm SEM of determinations on four to eight cultures; the total number of neurons assessed are indicated in parentheses to the right of each value.

${ }^{a} p<0.001$ compared to control.

${ }^{b} p<0.001$ compared to control or A23187.

c $p<0.05$ compared to control, $p<0.01$ compared to forskolin or dbcAMP + IBMX.

${ }^{d} p<0.01$ compared to reduced $\left[\mathrm{Ca}^{2+}\right]_{\mathrm{e}}$ 
about half of the neurons in the buccal population ceased outgrowth (Table 2). These data suggest that while cAMP can be an important regulator of neuronal outgrowth in a subpopulation of neuronal types, other cell types may use alternate mechanisms.

To determine if the suppressive effects of cAMP on the subpopulation of neurons sensitive to this messenger required calcium influx from the cell exterior, we exposed the total population of buccal neurons to forskolin in combination with a reduced calcium incubation medium. Prior to this final experiment, it was necessary to establish the effects of a reduced calcium medium alone on neurite outgrowth. Our previous results demonstrated that neurons B19 and B5 respond to a reduced calcium environment with continued elongation (Mattson and Kater, 1987; Fig. 8). However, when all neurons of the buccal ganglia were exposed to a reduced calcium medium, we found that neurite elongation was suppressed in approximately $20 \%$ of the neurons tested (Table 2). Variable degrees of growth cone motility were exhibited among the remaining $80 \%$ of the buccal neurons, which continued to elongate in the presence of a reduced calcium environment. Thus, we found a spectrum of outgrowth responses of neurons exposed to an environment expected to reduce calcium influx.

Since calcium influx was required for cAMP-induced suppression of neurite outgrowth from neurons B19 and B5 (Fig. 8), our final experiment tested the calcium dependency of cAMP's action on the subpopulation of cAMP-sensitive neurons (Table $2)$. When neurons were exposed to both forskolin $\left(10^{-4} \mathrm{M}\right)$ and the reduced calcium environment, outgrowth was suppressed in approximately $40 \%$ of neurons tested (Table 2). Since the percentage of neurons suppressed under the latter conditions was less than the number suppressed by forskolin alone, it appears that there are at least 2 subpopulations of forskolin-sensitive neurons, one which requires calcium influx for forskolin's action and one that is calcium-independent. Indeed, neurons B5 and B 19 can be catalogued in the cAMP-sensitive, calciumdependent subpopulation of buccal neurons.

The combined results suggest that while increases in calcium influx suppress neurite outgrowth in all neurons, elevations in cAMP affect only subsets of neurons within the buccal ganglion. Furthermore, cAMP may act independently of extracellular calcium or CAMP's action may require influx of calcium from the cell exterior depending upon the neuronal type.

\section{Discussion}

The data presented here encompass 3 main points. (1) The diverse neurons within even the relatively small population of the buccal ganglion apparently possess outgrowth response systems that may or may not respond to the same second messenger (cAMP). Indeed, cAMP-elevating agents inhibited the outgrowth of only half of the neurons in the buccal population. (2) Calcium appears to be a general regulator of ncuronal outgrowth. The data for ionophore A23187 indicate that all buccal neurons possess outgrowth response systems that can be stabilized by increases in calcium influx and are consistent with the hypothesis that calcium is a key regulator of growth cone motility and neurite elongation (Cohan et al., 1987; Mattson and Kater, 1987). (3) Depending upon the particular neuron, cAMP can affect neurite outgrowth independently of calcium influx or cAMP's action may require influx of calcium. In the 2 identified neurons studied here (B5 and B19) we found that cAMP suppression of outgrowth requires calcium influx. Given that among cAMP- sensitive neurons, only 1 in 4 requires calcium influx for suppression of outgrowth, it appears that neurons B19 and B5 are members of a relatively small subpopulation of buccal neurons whose cAMP outgrowth-regulating system is linked to influx of calcium. Finally, the data presented here indicate that in about one-third of the neurons in the buccal ganglion (those sensitive to both A23187 and cAMP-elevating agents in the absence of calcium influx), cAMP and calcium can suppress outgrowth by independent mechanisms. This heterogeneity among neurons in their intracellular outgrowth-regulating systems is not surprising considering the heterogeneity of these neurons with respect to morphology and function (Kater, 1985). Indeed, such diversity in second-messenger response systems for neuronal outgrowth may contribute to the generation of the diversity of neuronal morphologies in the nervous system.

\section{Intracellular mechanisms regulating neurite outgrowth: relationships to neurotransmitter actions}

In identified Helisoma neuron B19, 5-HT can suppress neurite outgrowth by increasing calcium influx through plasma membrane channels (Cohan et al., 1987; Mattson and Kater, 1987). In the present study we demonstrated that cAMP-elevating agents can mimick the suppression of outgrowth seen in response to 5-HT by a mechanism linked to calcium influx. Since cAMP is known to mediate the effects of neurotransmitters on calcium channel activity (see Kupfermann, 1980; Levitan, 1985, for reviews), it is reasonable to consider that 5-HT might use a similar cAMP-Ca ${ }^{2+}$ system to regulate neuronal outgrowth. Indeed, calcium channels can be directly activated by cAMP-dependent protein kinase in Aplysia bag cell neurons (Kaczmarek et al., 1980), while cAMP can block potassium channels and thus prolong a depolarization of the membrane with subsequent increased activation of voltage-sensitive calcium channels (Klein and Kandel, 1978; Grega et al., 1987). While the present study did not directly test the involvement of cAMP in 5-HT's action on neuron B19, several data sets are consistent with the hypothesis that 5-HT might use CAMP as a mediator of calcium influx in neurons B 19. Thus, the time courses of suppression of neurite outgrowth by both 5-HT (Mattson and Kater, 1987) and cAMP-elevating agents were indistinguishable. In addition, 5-HT causes influx of extracellular calcium into neuron B19 (Cohan et al., 1987). Finally, in neurons B5 and B19, cAMP's suppressive action required influx of calcium, a fact consistent with the possibility that cAMP induces calcium influx in these neurons. Verification of such a mechanism for regulation of neurite outgrowth by 5 -HT will require measurements of calcium currents under conditions of exposure to cAMP and biochemical analysis of intracellular cAMP levels in control neurons and neurons exposed to neurotransmitters.

In identified neurons B5 and B 19 cAMP does not appear to act on neurite outgrowth independent of the calcium system. Thus, suppression of outgrowth induced by the dbcAMP-IBMX (a combined treatment that circumvents adenylate cyclase and phosphodiesterase activities) is reversed by manipulations that reduce calcium influx, indicating that calcium is required for inhibition of outgrowth at a site distal to cAMP action. Notwithstanding the possibility that manipulations that reduce calcium influx across the plasma membrane may alter adenylate cyclase or phosphodiesterase activities and so change intracellular cAMP levels, it is clear that increases in intraneuronal calcium can act independently of cAMP to suppress outgrowth. Indeed, electrical activity as well as ionophore A23187, both of 
which stimulate calcium influx, suppressed outgrowth from all Helisoma neurons tested (Cohan and Kater, 1986; Cohan et al., 1987; Mattson and Kater, 1987). Thus, it appears that any mechanism that increases intracellular calcium levels can suppress neuronal outgrowth.

\section{Second-messenger systems affect neurite outgrowth differently} in different neurons

While identified neurons have provided a great deal of precision in analyses of the cellular bases of neuronal outgrowth (Kater and Mattson, 1988), the use of a larger population of unidentified buccal neurons allowed us to test whether the results obtained with the identified neurons applied to other neurons. The experiments in generic buccal neurons revealed that different neurons within the buccal population possess outgrowth response systems that are linked differentially to cAMP and calcium second-messenger systems. Thus, neurite outgrowth is inhibited by calcium influx in all neurons, while only $50 \%$ of the neurons are affected by elevations in cAMP. Furthermore, the action of cAMP is dependent upon calcium influx in a subpopulation of the CAMP-sensitive neurons. A calcium-independent mechanism of cAMP action appears to exist in approximately one-third of buccal neurons since cAMP suppressed outgrowth in these neurons in a manner not linked to calcium influx from the cell exterior. These results are consistent with the possibilities that calcium and cAMP can act independently or can interact to regulate the cytoskeletal elements that regulate neuronal outgrowth.

In mammalian neurons responsive to nerve growth factor, cAMP and calcium can both act as promoters of neurite outgrowth. In those cells, a cAMP-induced influx of calcium apparently mediates the outgrowth response (Schubert et al., 1978; Nirenberg et al., 1984). In contrast, Helisoma neurons B19 and B5 respond to either CAMP or calcium with progressive reductions in both neurite elongation and growth cone motility. It is clear from these opposite, neuron-specific effects on outgrowth of increases in cAMP and calcium that different neuronal types have outgrowth-response systems that respond differently to the same second messenger. We previously proposed that these opposite effects can be explained by postulating that the "calcium set-points" (i.e., basal levels of intracellular calcium of the neurons being studied) of the mammalian and Helisoma neurons are below and above, respectively, those optimum for neurite outgrowth (Mattson and Kater, 1987). Thus, while the cAMP and calcium systems in these 2 models appear to be similarly linked, the activation of the cAMP system in each neuronal type results in opposite effects on outgrowth. Our finding that the outgrowth from nearly $20 \%$ of buccal neurons is stabilized when the neurons are exposed to a reduced calcium environment indicates that, in addition to neuron-specific differences in calcium set points, there exist quantitative differences in calcium sensitivities. Thus, the moderate reductions in calcium influx expected with low levels of calcium channel blockers or reduced calcium mcdium can stop outgrowth in a small population of buccal neurons or accelerate outgrowth in others (neurons B19 and B5). The calcium set-points (Kater and Mattson, 1988; Mattson and Kater, 1987) of the neurons suppressed by reductions in calcium influx are likely to be in a very narrow optimum range for outgrowth, while those of the other $80 \%$ of buccal neurons not suppressed by reductions in calcium are likely to be above the optimum. In any case, it appears that, among diverse neuronal types, qualitative as well as quantitative dif- ferences exist in the second-messenger systems regulating outgrowth.

\section{Local control of growth cones by cAMP and calcium}

To date, the effects of all signals known to suppress neurite outgrowth (neurotransmitters, electrical activity, calcium) have been found to act locally on the growth cone proper. Similarly, we found that the suppression of growth cones by cAMP does not require the presence of the soma. It is therefore reasonable to consider that cAMP, acting through its kinase, can phosphorylate outgrowth-controlling substrates within the growth cone proper that mediate suppression of outgrowth. We do not know to what extent growth cones are regulated independently of the cell body during normal development in the intact nervous system. However, our analyses of the regulation of the isolated growth cone, taken together with the relative structural simplicity of this structure, indicate that the growth cone may prove a useful model for the study of second messenger regulation of cell motility.

The evolutionary process has conserved a scaffolding of cellular control systems to regulate a wide variety of cell processes. Onto such frameworks within individual neurons can be added diverse control mechanisms to build cellular individuality. The present findings in Helisoma neurons indicate that calcium may act as a common scaffold for the regulation of growth cone motility and neurite elongation. While adding support to the hypothesis that calcium is a general regulator of neuronal outgrowth (Mattson and Kater, 1987), our findings also demonstrate that there is considerable heterogeneity of second-messenger response systems among neuronal subtypes with respect to regulation of neurite outgrowth by cAMP. Thus, cAMP can be considered as a modifier of the calcium framework. An additional second messenger system that we have not addressed in the present study is the inositol phospholipid-protein kinase C system (Nishizuka, 1984), which has been shown to affect neuronal outgrowth in other cell types (Ishii, 1978; Spinelli and Ishii, 1983; Traynor, 1984; Traynor and Schubert, 1984; Hama et al., 1986). Thus, in addition to differences among neuronal types in the roles of cAMP and calcium in regulating neurite outgrowth, the inositol phospholipid system is likely to add further complexity. It thus appears that it will be necessary to work out individual schema of second-messenger interactions involved in the regulation of neurite outgrowth for particular neuronal systems and probably for neuronal subtypes and even individual identified neurons.

\section{References}

Anglister L., I. C. Farber, A. Shahar, and A. Grinvald (1982) Localization of voltage-sensitive calcium channels along developing neurites: Their possible role in regulating neurite elongation. Dev. Biol. 91: 351-365.

Berridge, M. (1984) Inositol triphosphate and diacylglycerol as second messengers. Biochem. J. 220: 345-354.

Bixby, J. L., and N. C. Spitzer (1984) Early differentiation of vertebrate spinal neurons in the absence of voltage-dependent $\mathrm{Ca}^{2+}$ and $\mathrm{Na}^{+}$ influx. Dev. Biol. 106: 89-96.

Bray, D., and D. Gilbert (1981) Cytoskeletal elements in neurons. Annu. Rev. Ncurosci. 4: 505-523.

Cohan, C. S., and S. B. Kater (1986) Suppression of neurite elongation and growth cone dynamics by electrical activity. Science 232 : 16381640 .

Cohan, C. S., P. G. Haydon, and S. B. Kater (1985) Single channel activity differs in growing and nongrowing growth cones of isolated identified neurons of Helisoma. J. Neurosci. Res. 13: 285-300.

Cohan, C. S., J. A. Connor, and S. B. Kater (1987) Electrically and 
chemically mediated increases in intracellular calcium in neuronal growth cones. J. Neurosci. 7: 3588-3599.

Connor, J. A., and P. Hockberger (1985) Calcium and cyclic AMP: Second messengers in gastropod neurons. In Model Neural Networks and Behavior, J. A. Selverston, ed., Plenum, New York.

Daly, J. W., W. Padgett, and K. B. Seamon (1982) Activation of cyclic AMP-generating systems in brain membranes and slices by the diterpene forskolin: Augmentation of receptor-mediated responses. J. Neurochem. 38: 532-544.

Doroshenko, P. A., P. G. Kostyuk, A. E. Martynynyuk, M. D. Kursky, and Z. D. Vorobetz (1984) Intracellular protein kinase and calcium inward currents in perfused neurons of the snail Helix pomatia. Neuroscience 11: 263-267.

Goldberg, J. I., P. Natvig, and S. B. Kater (1986) Embryonic and postembryonic development of the serotonin phenotype in Helisoma. Soc. Neurosci. Abstr. 12: 1163.

Greene, L. A., and E. M. Shooter (1980) The nerve growth factor. Annu. Rev. Neurosci. 3: 353-405.

Greengard, P. (1978) Phosphorylated proteins as physiological effectors. Science 199: 146-152.

Grega, D. S., M. A. Werz, and R. L. MacDonald (1987) Forskolin and phorbol esters reduce the same potassium conductance of mouse neurons in culture. Science 235: 345-348.

Hama, T. K., P. Huang, and G. Guroff (1986) Protein kinase C as a component of a nerve growth factor-sensitive phosphorylation system in PC12 cells. Proc. Natl. Acad. Sci. USA 83: 2353-2357.

Haydon, P. G., D. P. McCobb, and S. B. Kater (1984) Serotonin selectively inhibits growth cone dynamics and synaptogenesis of specific identified neurons. Science 226: 561-564.

Haydon, P. G., C. S. Cohan, D. P. McCobb, H. R. Miller, and S. B. Kater (1985) Neuron-specific growth cone properties as seen in identified neurons of Helisoma. J. Neurosci. Res. 13: 135-147.

Ishii, D. N. (1978) - Effect of tumor promoters on the response of cultured embryonic chick ganglia to nerve growth factor. Cancer Res. 38: 3886-3893.

Kaczmarek, L. K., K. R. Jennings, F. Strumwasser, A. C. Nairn, U. Walter, F. D. Wilson, and P. Greengard (1980) Microinjection of catalytic subunit of cyclic AMP-dependent protein kinase enhances calcium action potentials of bag cell neurons in cell culture. Proc. Natl. Acad. Sci. USA 77: 7487-7491.

Kater, S. B. (1985) Dynamic regulators of neuronal form and connectivity in the adult snail Helisoma. In Model Neural Networks and Behavior, J. A. Selverston, ed., Plenum, New York.

Kater, S. B., and M. P. Mattson (1988) Extrinsic and intrinsic regulators of neurite outgrowth and synaptogenesis in isolated, identified Helisoma neurons in culture. In Cell Culture Approaches to Invertebrate Neuroscience, D. J. Beadle and S. B. Kater, eds., Academic, London (in press).

Klein, M., and E. R. Kandel (1978) Presynaptic modulation of voltagedependent $\mathrm{Ca}^{2+}$ currents: Mechanism for behavioral sensitization in Aplysia californica. Proc. Natl. Acad. Sci. USA 75: 3512-3516.

Kostenko, M. A., V. S. Musienko, and T. I. Smolikhina (1983) $\mathrm{Ca}^{2+}$ and $\mathrm{pH}$ affect the neurite formation in cultured mollusc isolated neurons. Brain Res. 276: 43-50.

Kupfermann, I. (1980) Role of cyclic AMP in excitable cells. Annu. Rev. Physiol. 42: 629-641.
Letourneau, P. C., and N. K. Wessells (1974) Migratory cell locomotion versus nerve axon elongation. Differences based on the effects of lanthanum ion. J. Cell. Biol. 61: 56-69.

Levitan, I. B. (1985) Phosphorylation of ion channels. J. Membrane Biol. 87: 177-190.

Mattson, M. P., and S. B. Kater (1987) Calcium regulation of neurite elongation and growth cone motility. 7: 4034-4043.

Mattson, M. P., and E. Spaziani (1986) Calcium antagonizes cAMPmediated suppression of crab Y-organ steroidogenesis in vitro: Evidence for activation of cAMP-phosphodiesterase by calcium-calmodulin. Mol. Cell Endocrinol. 48: 135-151.

McCobb, D. P., P. G. Haydon, and S. B. Kater (1985) Dopamine: An additional regulator of neurite outgrowth in Helisoma. Soc. Neurosci. Abstr. 11: 761 .

Nirenberg, M., S. Wilson, H. Higashida, A. Rotter, K. Krueger, N. Busis, R. Ray, J. G. Kenimer, and M. Adler (1984) Modulation of synapse formation by cyclic adenosine monophosphate. Science 222: 794 799.

Nishizuka, Y. (1984) Turnover of inositol phospholipids and signal transduction. Science 225: 1365-1372.

Rasmussen, H., and P. Q. Barrett (1984) Calcium messenger system: An integrated view. Physiol. J. (Lond.) 64: 938-984.

Reboulleau, C. P. (1986) Extracellular calcium-induced neuroblastoma differentiation: Involvement of phosphatidylinositol turnover. J. Neurochem. 46: 920-930.

Reed, P. W., and H. A. Lardy (1972) A23187: A divalent cation ionophore. J. Biol. Chem. 247: 6970-6977.

Schubert, D., M. LaCorbiere, C. Whitlock, and W. Stallcup (1978) Alterations in the surface properties of cells responsive to nerve growth factor. Nature 273: 718-723.

Shapiro, D. L. (1973) Morphological and biochemical alterations in foetal rat brain cells cultured in the presence of monobutyryl cyclic AMP. Nature 241: 203-204.

Spinelli, W., and D. N. Ishii (1983) Tumor promoter receptor regulation of neurite formation in cultured human neuroblastoma cells. Cancer. Res. 43: 4119-4125.

Suarez-Isla, B. A., D. J. Pelto, J. M. Thompson, and S. I. Rapoport (1984) Blockers of calcium permeability inhibit neurite extension and formation of neuromuscular synapses in cell culture. Dev. Brain Res. 14: 263-270.

Sutherland, E. W. (1972) Studies on the mechanism of hormone action. Science 177: 401-406.

Traynor, A. E. (1984) The relationship between neurite extension and phospholipid metabolism in PC12 cells. Dev. Brain Res. 14: 205210.

Traynor, A. E., and D. Schubert (1984) Phospholipases elevate cyclic AMP levels and promote neurite extension in a clonal nerve cell line. Dev. Brain Res. 14: 197-204.

Wong, R. G., R. D. Hadley, S. B. Kater, and G. C. Hauser (1981) Neurite outgrowth in molluscan organ and cell cultures: The role of conditioning factor(s). J. Neurosci. 1: 1008-1021.

Wong, R. G., D. L. Barker, S. B. Kater, and D. A. Bodnar (1984) Nerve growth-promoting factor produced in culture media conditioned by specific CNS tissues of the snail Helisoma. Brain Res. 292: $81-91$. 